\title{
Ataxia due to vitamin E deficiency
}

\author{
H. Rahmoune', N. Boutrid'. \\ ${ }^{1}$ Pediatrics Dept., University Hospital of Setif - Setif 1 University, Algeria
}

\section{INTRODUCTION}

The large spectrum of herediatary ataxias include some peculiar etiologies like isolated vitamin E deficiency, a specific and treatable cause in children.

Our poster is the anamnestic, clinical and biological features of this particular disorder in two siblings.

\section{MATERIAL \& METHODS}

A 5 years old girl presents progressive ataxia and dysarthria, with diffuse hyporflexia/areflexia since 12 months . Her somatic and neurological return normal , excpeting theses symptoms.

Her brother, aged 2 years, also exhibits right hyporeflexia and his mother reports several spontaneous falls.

Frataxin gene sequencing is negative, and serum vitamin $\mathrm{E}$ is very diminished, even unmeasurable according to the laboratory reference ranges.

\section{RESULTATS}

In fact, these two siblings have the peculiar Ataxia with vitamin E deficiency. They started $1000 \mathrm{mg} / \mathrm{day}$ of liposoluble capsule of vitamin E, and showed impressive improvement within 3 months ; specially in their tendon reflexes and night walking balance.

Unfortunately, genetic test of the tocopherol transfer protein (TTP) gene could not been performed.

\section{DISCUSSION}

Ataxia with vitamin E deficiency (AVED) is very peculiar : it generally manifests in late childhood or early teens with dysarthria, poor balance when walking (especially in the dark), and progressive clumsiness.

AVED is caused by mutations in the (TTPA; 8q13). This protein binds alpha-tocopherol (a vitamin E isomer) and very-low-density lipoproteins (VLDLs) in the liver. When mutated, TTPA prevents vitamin E linking to VLDLs, preventing it to pass into general circulation.

The majority of the reported cases originated in the Mediterranean region, and the 744delA mutation is the most common.

AVED is confirmed by a simple blood sample (normal lipids and liporproteins but very low vitamin E), along with a definitive exclusion of Friedreich ataxia, the close form of such ataxia. (7)

Treatment is based on continuous, daily supplements of vitamin E as high as 800-1500 mg/day.

\section{CONCLUSION}

AVED is frequently reported in the Mediterranean area and responds to dietary supplementation.

Every child with ataxia should be tested to make an early diagnosis, start vitamin $E$ 